\title{
Evaluation of Bacterial Load and Antibiotic Resistance Pattern of Staphylococcus aureus from Ready-to-Eat Raw Beef in Bahir Dar City
}

Bizuneh Tsehayneh ( $\sim$ mbalew2@gmail.com )

Mizan Agricultural Technical Vocational and Educational Training College

Taddesse Yayeh

Bahir Dar University College of Agriculture and Environmental Sciences

Birhan Agmas

Bahir Dar University College of Agriculture and Environmental Sciences

Semahegn Yilkal

Bahir Dar University College of Agriculture and Environmental Sciences

Research article

Keywords: Antibiotic; Bahir Dar; Beef; Butcher shops; Microbial load; Staphylococcus aureus

Posted Date: August 21st, 2019

DOI: https://doi.org/10.21203/rs.2.13268/v1

License: (a) (i) This work is licensed under a Creative Commons Attribution 4.0 International License.

Read Full License

Version of Record: A version of this preprint was published at International Journal of Microbiology on March 25th, 2021. See the published version at https://doi.org/10.1155/2021/5560596. 


\section{Abstract}

Background Staphylococcus aureus is one of the most important causes of foodborne intoxication and most frequent antibiotic resistant pathogen in the world. Regular evaluation of the current safety status of food is a pro-active measure to minimize the possible danger of foodborne pathogens. Therefore, this study was conducted on to assess the bacterial load and antibiotic resistance profile of $\mathrm{S}$. aureus from ready-to-eat raw beef in Bahir Dar city. Methodology This cross-sectional study was conducted from October 2018 to April 2019 by collecting a total 101 raw beef samples from butcher shops using simple random sampling method. Isolation and microbial load determination of S. aureus using was performed conventional culture method as well as antibiotic susceptibility test was conducted by using Kirby Bauer disk diffusion method on Mueller-Hinton agar. The data were analyzed by using STATA software version 12.0. Result Out of 101 raw beef samples, $55(54.45 \%)$ were positive for $S$. aureus with mean bacterial count of $3.40 \pm 0.63(\log 10 \mathrm{cfu} / \mathrm{g})$. About $13 \%$ of butcher shops had unacceptable and potentially dangerous (above 104cfu/g) bacterial load. High S. aureus drug resistance were observed on penicillin $(92.73 \%)$ followed by cefoxitin (74.5\%), tetracycline (63.63\%), clindamycin (50.9\%). On the other hand there were highest susceptibility for ciprofloxacin (100\%) followed by gentamycin $(90.91 \%)$ and erythromycin (87.27\%). Multi-drug resistance was also found in $54(98 \%)$ of the isolates. Conclusion In this study highly drug resistant $\mathrm{S}$. aureus was a major meat contaminant in butcheries of Bahir Dar city. Therefore, appropriate antimicrobial use and staphylococcal control methods should be employed to prevent S. aureus intoxications in foods.

\section{Background}

Animal origin food items are rich sources of nutrients and provide a variety of micronutrients to humans that are not obtained in plant-derived foods [1]. Meat is one of the most nutritive and favorite animalsource foods. Due to its high water content ( 0.99 water activity), rich in proteins, minerals and other nutrients which are suitable for microbial growth, meat is highly perishable food that can cause infection in humans and also can lead to economic loss due to spoilage $[2,3]$.

A large number of foodborne zoonotic diseases often occur due to the consumption of contaminated animal origin foods such as meat and milk [4]. Among the bacteria predominantly involved in foodborne diseases (FBD), S. aureus is one of the leading causes of foodborne intoxication throughout the world resulting from the consumption of preformed staphylococcal enterotoxins [5-7]. Due to poor hygienic practices and low level of awareness, this problem is worse in developing countries.

Staphylococcal foodborne intoxication (SFI) is often associated with the ingestion of highly heat stable staphylococcal enterotoxins. The acceptable level of $S$. aureus in ready-to-eat food should be below $10^{3}$ colony forming unit per gram (cfu/g) of food. If the amount of bacteria is greater than $10^{4} \mathrm{cfu} / \mathrm{g}$, the food is unsatisfactory and potentially hazardous for health and/or unfit for human consumption [8]. Ingestion of nanogram to a microgram of staphylococcal enterotoxin contaminated food can cause serious illness ranging from minor skin infection to life threatening diseases [9]. 
Antimicrobial resistance is a serious threat to public health across the globe. A wide variety of antimicrobial drugs are employed to treat $S$. aureus infections. However, emergence and spread of antimicrobial resistant $S$. aureus isolates constitute a global challenge for the effective treatment and control of these infections [10-12].

In Ethiopia, the burden and public health impact of foodborne illness related with $S$. aureus infection is poorly understood. However, the epidemiology of this bacteria and the widespread habit of raw meat and milk consumption in the population are suggestive of the risk of acquiring $S$. aureus [13]. Consuming raw beef in the form of simple cut strips of meat which is locally called "Kurt", is a common habit and is an indication of wealth in Ethiopia[14]. Moreover, raw beef is available in open-air local butchers of Ethiopia without the cold-chain process which could be serving as a potential source for foodborne illnesses [15, 16].

Besides the prevalence of $S$. aureus in meat reaches up to $40 \%$ [17] in butcher shops of Mekelle city, there is a paucity of data regarding to $S$. aureus from ready-to-eat raw beef in Bahir Dar city. Therefore, this study was focused on isolation and identification of $S$. aureus, measuring its magnitude and antibiotic resistance pattern from ready-to-eat raw beef to provide useful information regarding to staphylococcal loads and their antibiotic resistance profile in Bahir Dar city.

\section{Methods}

\section{Study area and study design}

The current cross-sectional study was conducted from October 2018 to April 2019 in Bahir Dar city (located $565 \mathrm{~km}$ north-northwest of Addis Ababa) in ready-to-eat raw beef retailers of butcheries. Geographically Bahir Dar is located between $11.29^{\circ}$ to $11.38^{\circ}$ North latitude and $37.23^{\circ}$ to $37.36^{\circ}$ East longitude. Its average elevation is estimated to be 1810 meter above sea level. The city's mean annual temperature ranges from $7.1^{\circ} \mathrm{C}$ to $29.7^{\circ} \mathrm{C}$, whereas annual mean temperature was $20.85^{\circ} \mathrm{C}$ [18].

\section{Sample size determination and sampling procedures}

In Bahir Dar city, about 137 licensed butcher shops were operating on meat and meat products and all the butcheries were included in the sampling procedure. The lists of all butcher shops were obtained from the health centers. The sample size were calculated by using Thrusfield formula for small sampling population[19] and a total of 101 retailers were selected based on simple random sampling.

\section{Data collection}

From randomly selected butcher shops, about 250 gram of ready-to-eat raw beef (Kurt) samples were collected in sterile stomacher plastic bags and kept in icebox containing ice. The collected samples were immediately taken to Bahir Dar University, Institute of Technology food microbiology laboratory for homogenization and the homogenate were transported to the Amhara Public Health Institute (APHI) microbiology laboratory unit within 4 hours by keeping the cold chain for bacteriological analysis. 


\section{Bacteriological investigation}

Isolation and identification of $S$. aureus from ready-to-eat raw beef was done according the methods described by ISO 6888-3 [20] and [21]. Briefly 25 gram of raw beef sample was transferred aseptically into a sterile stomacher bag containing $225 \mathrm{ml}$ of peptone water and homogenized for 3 minute using a stomacher. From the original homogenate, a loopful aliquot was spread on mannitol salt agar (MSA) and incubated from $24-48$ hours at $37^{\circ} \mathrm{C}$. Due to high salt concentration of MSA, it may not support the growth of weak and injured bacteria during sample processing and may result to false negative growth. In order to avoid this problem, $50 \mathrm{ml}$ of the original homogenate was directly incubated for $24-48$ hours at $37^{\circ} \mathrm{C}$ and a loopful of inoculum from enrichment broth was also cultured on MSA. Pure cultures of presumptive colonies (yellow colonies on MSA) were streaked on nutrient agar and incubated for 24-36h at $37^{\circ} \mathrm{C}$ for Gram stain and further biochemical tests (catalase, coagulase test and oxidationfermentation test). In addition, presumptive colonies was also inoculated to blood agar plates (5\% difibrinated sheep blood) and plates were incubated aerobically at $37^{\circ} \mathrm{C}$ and examined after 24 hours of incubation for growth and hemolytic pattern of $S$. aureus.

\section{Enumeration of Staphylococcus aureus count}

In addition to identification, microbial counts of $S$. aureus were conducted on MSA by using spread plate count method. After confirming the sample was positive for $S$. aureus, tenfold of serial dilutions from the original homogenate were prepared and a $0.1 \mathrm{ml}$ sample from serial dilutions was spread on MSA and incubated from $24-48$ hours at $37^{\circ} \mathrm{C}$. Presumptive colonies were undergoing confirmatory test and golden yellow colonies on MSA with catalase and coagulase positive isolates, and complete hemolysis on blood agar were identified as $S$. aureus ount and the number of $\mathrm{cfu} / \mathrm{g}$ of the test sample was calculated by the formula as described below $[22,23]$.

cfu per gram of sample $=c / d^{*} v$

Where:- $c=$ is the number of colonies on the counted plate,

$d=$ the dilution rate of the counted plate and

$v=$ the inoculated volume of this dilution

\section{Anti-microbial susceptibility testing}

All positive isolates of $S$. aureus were subjected to antibiotic susceptibility test by using the Kirby Bauer disc diffusion method as per Clinical Laboratory Standards Institute (CLSI) of USA guidelines on MuellerHinton agar (MHA). The antibiotics were selected based on the groupings of antibiotic agents with United States Food and Drug Administration clinical indications that should be considered for routine testing and reporting on non-fastidious organisms. One representative antibiotic agent from each subclass of antibiotics groups, commonly used and most available antibiotics for treatment of staphylococcal related diseases in animal and human was selected. Based on the above criteria, 9 antibiotics [chloramphenicol 
$(30 \mu \mathrm{g})$, ciprofloxacin $(5 \mu \mathrm{g})$, cefoxitin $(30 \mu \mathrm{g})$, clindamycin $(2 \mu \mathrm{g})$, erythromycin $(15 \mu \mathrm{g})$, gentamycin $(10 \mu \mathrm{g})$, penicillin (10 units), tetracycline $(30 \mu \mathrm{g})$ and trimethoprim-sulfamethoxazole $(1.25 / 23.75 \mu \mathrm{g})]$ were selected for this study [24].

For susceptibility test, three to five well-isolated colonies of the same morphological type were selected from nutrient agar plate culture and transferred into test tubes containing sterile saline and mixed thoroughly. The density of the suspension was adjusted to McFarland 0.5 by addition of saline or more $S$. aureus colony. A sterile swab was dipped into the suspension and the excess of inoculums were removed by pressing it against the sides of the tube to avoid over-inoculation of plates. The inoculums were spread evenly over the entire surface of the agar plate by swabbing in three directions. Antibiotic discs were applied firmly on the agar surface and incubated for $24 \mathrm{~h}$ at $37^{\circ} \mathrm{C}$. The diameter of the zone of inhibition around the disc was measured using ruler in millimeter $(\mathrm{mm})$ and interpreted according to the standard of CLSI as susceptible, intermediate or resistant [24-26]. Those isolates showing resistance to three or more antibiotics were considered as multiple drug resistant (MDR) [20].

\section{Data quality assurance}

The data quality and the reliability of the study findings were assured by following standard operating procedures and the routine use of control bacterial strains. The sterility of prepared media was checked by incubating some randomly selected plates for $24-48$ hours at $37^{\circ} \mathrm{C}$. Uninoculated media was incubated as negative control to check for sterility. The quality of the culture media and test procedures were thoroughly checked using standard American Type Culture Collection (ATCC) strain of S. aureus (ATCC25923) as a positive control for screening tests, confirmatory tests and disk diffusion antibiotic susceptibility tests. Escherichia coli ATCC-25922 was used as a negative control for culture on mannitol salt agar.

\section{Data management and statistical analysis}

Raw data and laboratory findings were encoded into Microsoft Excel, exported into STATA software version 12.0 and analyzed using descriptive statistics such as frequency, percentages, mean and standard deviation (SD). In all the analyses, confidence level was held at $95 \%$ and $p$-value was assumed less than $5 \%(P<0.05)$.

\section{Results And Discussion}

\section{Isolation rate of Staphylococcus aureus}

In the present investigation, from a total of 101 ready-to-eat raw beef samples subjected for cultural and biochemical isolation, 55(54.45\%) were positive for S. aureus which suggests that the bacteria could be a major food contaminant in butcher's shops of Bahir Dar city.

\section{Microbial load of Staphylococcus aureus}


The enumeration of $S$. aureus was performed on MSA using spread plate technique. The minimum and maximum S. aureus counts in ready-to-eat raw beef samples of Bahir Dar city were 2.48 and 5.08 $\left(\log _{10} \mathrm{cfu} / \mathrm{g}\right)$ respectively with a mean and standard deviation of $3.40 \pm 0.63\left(\log _{10} \mathrm{cfu} / \mathrm{g}\right)$. Based on CFS (2014) microbiological guidelines for food, none of the butcher shops had good microbial level (below $100 \mathrm{cfu} / \mathrm{g}$ ) and majority of them (49\%) had unsatisfactory level followed by acceptable microbial level (38\%) for S. aureus counts (Figure 1). The mean count of $S$. aureus in this study was in line with $3.88 \log 10 \mathrm{cfu} / \mathrm{g}$ reported from fresh meat Bahir Dar City [27]. About $13 \%$ of butcher shops had unacceptable and potentially dangerous bacterial load. If the bacterial count exceeds the above standard in fresh meat, then the meat is not acceptable and this indicates alarm signals on meat hygiene along meat chain from abattoir to butcher shops [28].

The mean count of $S$. aureus this study was lower than the findings $\left(5.61 \pm 0.10 \log 10 \mathrm{cfu} / \mathrm{cm}^{2}\right.$ for cutting boards and $6.43 \pm 0.34 \log 10 \mathrm{cfu} / \mathrm{cm}^{2}$ for butchers' hands) from retail houses of Jigjiga town [29]. These variations of bacterial load might be due the difference of meat processing, handling practices and sanitary standard operating procedures along the meat production chain.

In most of the retail points, meats were seen on the ground and left to the mercies of the environment which can create an avenue for microbial pathogens to proliferate on it. These high bacterial loads could affect the average shelf life of the meat and increase the chances of spoilage.

\section{Antibiotic susceptibility profile of Staphylococcus aureus}

All the 55 positive samples of $S$. aureus isolates were undergo in-vitro antibiotic susceptibility test on MHA medium by using disc diffusion technique. The isolates were completely susceptible to ciprofloxacin followed by gentamycin (89.09\%) (Table 1). This finding was agreed with the finding that all $S$. aureus isolates from meat sample in Addis Ababa were susceptible (100\%) to ciprofloxacin [30]. This finding was also compatible with the previous research output from dairy farm and abattoir in Addis Ababa that $97.7 \%$ of the isolates were susceptible to ciprofloxacin [20]. The reason for ciprofloxacin susceptibility of the isolates could be due to the fact that ciprofloxacin is relatively expensive and newly introduced antibiotic as compared to the other common antibiotics [27].

Majority of the bacterial isolates $(92.73 \%$ ) were found to be resistant to the antibiotic penicillin and $74.5 \%$ of the isolates were resistant for cefoxitin. The present study was in harmony with report indicated that $95.3 \%$ of the isolates were resistant to penicillin [20]. The reason for high resistance of penicillin and other $\beta$-lactam antibiotics could be that they are the most commonly used antibiotics for the treatment of infection in humans and animals in Ethiopia [20].

Table 1. Antibiotic susceptibility pattern of $S$. aureus isolates from raw beef sample in Bahir Dar city $(\mathrm{N}=55)$ 


\begin{tabular}{llll}
\hline Antibiotic Agent & \multicolumn{3}{l}{ Response of S aureus for antibiotics N(\%) } \\
& Susceptible & Intermediate & Resistant \\
\hline Chloramphenicol & $44(80)$ & $2(3.63)$ & $9(16.36)$ \\
Ciprofloxacin & $55(100)$ & - & - \\
\hline Cefoxitin & $14(25.45)$ & - & $41(74.5)$ \\
\hline Clindamycin & $22(40)$ & $5(9.09)$ & $28(50.9)$ \\
\hline Erythromycin & $40(72.72)$ & $8(14.55)$ & $7(12.73)$ \\
\hline Gentamycin & $49(89.09)$ & $1(1.81)$ & $5(9.09)$ \\
\hline Penicillin & $4(7.27)$ & - & $51(92.73)$ \\
\hline Tetracycline & $16(29.09)$ & $4(7.27)$ & $35(63.63)$ \\
\hline Trimethoprim-sulfamethoxazole & $39(70.9)$ & $3(5.45)$ & $13(23.63)$ \\
\hline
\end{tabular}

According to the principles of CLSI, the susceptibility or resistance results of cefoxitin can be applied to methicillin [24]. In this research $74.5 \%$ isolates of $S$. aureus were resistant to cefoxitin that considered to be MRSA and out of the 41 cefoxitin resistant isolates, 36 (87.80\%) were also resistant to penicillin. This could be due to the fact that, the resistance of $S$. aureus to those drugs might be attributed to the presence of the mecA gene which has low affinity for $\beta$-lactam antibiotics [31, 32].

The present study revealed that about $98 \%$ of $S$. aureus isolates had high resistance to two or more drugs due to the fact that there is frequent irrational antimicrobial use and misuse behavior in the country [20] (Figure 2).

\section{Multidrug resistance profile of $S$. aureus}

From the tested 55 isolates of $S$. aureus, 46 (83.64\%) isolates showed MDR. Nineteen isolates were resistant to three antibiotics, similarly twenty and seven isolates were resistant to four and five antibiotics respectively (Table 2). The presence of MDR of $S$. aureus isolates in this study area indicates the possible significant risk of the resistant strain along the studied beef line.

Table 2. Multiple drug-resistance profile of $S$. aureus isolates from raw beef sample in Bahir Dar city 


\begin{tabular}{|l|l|l|}
\hline $\begin{array}{l}\text { Number of antibiotics } \\
\text { showing resistance }\end{array}$ & $\begin{array}{l}\text { Number and frequency (\%) of } \\
\text { resistant isolates }\end{array}$ & Type of antibiotics \\
\hline Three & $19(34.54 \%)$ & CAF+CLI+PEN \\
& & CEF+CAF+PEN \\
CEF+CLI+PEN (4) & CEF+ERY+PEN \\
& & CEF+ERY+TTC \\
& & CEF+GEN+PEN \\
& & CEF+PEN+TSX \\
& & CEF+PEN+TTC (5) \\
& & CLI+PEN+TSX \\
& PEN+TTC+TSX (3) \\
\hline Four & $20(36.36)$ & CAF+CEF+CLI+PEN \\
& & CAF+CEF+PEN+TTC (2) \\
& & CAF+CLI+PEN+TTC \\
& & CEF+CLI+ERY+TSX (2) \\
& & CEF+CLI+PEN+TSX \\
& & CEF+CLI+PEN+TTC (9) \\
& & CEF+PEN+TTC+TSX (2) \\
& & CLI+PEN+TTC+TSX \\
\hline Five & & GEN+PEN+TTC+TSX \\
\hline & & CAF+CEF+CLI+PEN+TTC \\
& & CEF+CLI+ERY+PEN+TTC \\
& & CEF+CLI+GEN+PEN+TTC \\
& & (2) \\
& & CEF+GEN+PEN+TTC+TSX \\
\hline
\end{tabular}

$\mathrm{CAF}=$ chloramphenicol, $\mathrm{CEF}=$ cefoxitin, $\mathrm{CLI}=$ clindamycin, $\mathrm{ERY}=$ erythromycin, GEN=gentamycin, $\mathrm{PEN}=$ penicillin, TTC=tetracycline, TSX=trimethoprim-sulfamethoxazole

\section{Conclusions And Recommendations}

This study revealed that $S$. aureus was a major contaminant of ready-to-eat raw meat in Bahir Dar city and nearly two third of contaminated beef samples had above the acceptable threshold level of bacterial load. Of all tested antibiotics, all $S$. aureus isolates were totally susceptible to ciprofloxacin. On the other hand, the highest resistance was observed to penicillin followed by cefoxitin. Almost all of the isolates were developed MDR and about half of them showed resistance to four or more classes of antibiotics suggesting wide distribution of MDR $S$. aureus stains from ready-to-eat beef existed. This may pose a risk to beef consumers due to the likelihood of food intoxication and antibiotic resistance problems.

Therefore, appropriate $S$. aureus control strategies should be designed and implemented, staphylococcal infections due to $S$. aureus is better to be treated using most potent antibiotics like ciprofloxacin and gentamycin with proper antimicrobial usage. Conducting further research regarding to antibiotic resistance genes and enterotoxin genes on $S$. aureus is advisable.

\section{Declarations}




\section{Ethical approval and consent}

The study was conducted after the protocol was ethically reviewed and approved by Institutional Review Board of Bahir Dar University, College of Agriculture and Environmental Sciences, School of Animal Production and Veterinary Medicine. A letter of support from Bahir Dar University was written to APHI. Then, ethical clearance was obtained from APHI and official permission was received from Bahir Dar city Administration Health Bureau.

Butchery's owner permission was asked to take raw beef sample with full consent by explaining the objectives of the study, their right and their participation is fully voluntarily. To ensure confidentiality, any personal or butchery identifying information was not collected and it was managed with unique code.

\section{Availability of data and materials}

The datasets used and/or analysed during the current study are available from the corresponding author on reasonable request.

\section{Competing interests}

The authors declare that they have no competing interests.

\section{Funding}

The fund for this study was obtained from Mizan Agricultural Technical Vocational and Educational Training College and Bahir Dar University

\section{Authors' contributions}

BT developed research idea and conducted the main study. TA and BA supervised, commented and corrected the research protocol, data analysis and all the write-up processes. SY had major contribution during data collection and laboratory investigation. All authors read and approved the final manuscript.

\section{Acknowledgements}

As per their contribution to this study, stuff members of Mizan Agricultural Technical Vocational and Educational Training College and Bahir Dar University are acknowledged. The support of laboratory technicians of Amhara Public Health Institute and Bahir Dar University Technology Institute, and butcher shop owners of Bahir Dar city is highly appreciated.

\section{References}

1. Popkin BM, Du S: Dynamics of the nutrition transition toward the animal foods sector in China and its implications: a worried perspective. The Journal of nutrition 2003, 133(11):3898S-3906S. 
2. Fratamico PM, Bhunia AK, Smith JL: Foodborne pathogens: microbiology and molecular biology: Horizon Scientific Press; 2005.

3. Thanigaivel G, Anandhan AS: Isolation and characterization of microorganisms from raw meat obtained from different market places in and around Chennai. Journal of Pharmaceutical, Chemical and Biological Sciences 2015, 3(2):295-301.

4. Sanyaolu A, Okorie C, Mehraban N, Ayodele O, Tshitenge S: Epidemiology of Zoonotic Diseases in the United States: a comprehensive review. J Infect Dis Epidemiol 2016, 2(021):10.23937.

5. Balaban N, Rasooly A: Staphylococcal enterotoxins. International journal of food microbiology 2000, 61(1):1-10.

6. Le Loir Y, Baron F, Gautier M: Staphylococcus aureus and food poisoning. Genet Mol Res 2003, 2(1):63-76.

7. Behling RG, Eifert J, Erickson MC, Gurtler JB, Kornacki JL, Line E, Radcliff R, Ryser ET, Stawick B, Yan Z: Selected pathogens of concern to industrial food processors: infectious, toxigenic, toxicoinfectious, selected emerging pathogenic bacteria. In: Principles of microbiological troubleshooting in the industrial food processing environment. Springer; 2010: 5-61.

8. CFS: Centre for Food Safety: Microbiological Guidelines for Food (for ready-to-eat food in general and specific food items). . In: Food and Environmental Hygiene Department. vol. 43/F. Queensway Government Offices, Hong Kong; 2014: 12.

9. Seo KS, Bohach GA: Staphylococcus aureus. In: Food Microbiology: Fundamentals and Frontiers, Third Edition. American Society of Microbiology; 2007: 493-518.

10. Geenen PL, Koene M, Blaak H, Havelaar AH, Van de Giessen A: Risk profile on antimicrobial resistance transmissible from food animals to humans. 2011.

11. Argudín M, Deplano A, Meghraoui A, Dodemont M, Heinrichs A, Denis O, Nonhoff C, Roisin S: Bacteria from animals as a pool of antimicrobial resistance genes. Antibiotics 2017, 6(2):12.

12. Yılmaz EŞ, Aslantaş Ö: Antimicrobial resistance and underlying mechanisms in Staphylococcus aureus isolates. Asian Pacific journal of tropical medicine 2017, 10(11):1059-1064.

13. Tarekgne E, Skeie S, Rudi K, Skjerdal T, Narvhus JA: Staphylococcus aureus and other Staphylococcus species in milk and milk products from Tigray region, Northern Ethiopia. African Journal of Food Science 2015, 9(12):567-576.

14. Wabeto W, Abraham Y, Anjulo AA: Detection and identification of antimicrobial-resistant Salmonella in raw beef at Wolaita Sodo municipal abattoir, Southern Ethiopia. Journal of Health, Population and Nutrition 2017, 36(1):52.

15. Ejeta G, Molla B, Alemayehu D, Muckle C: Salmonella serotypes isolated from minced meat beef, mutton and pork in Addis Ababa, Ethiopia. Revue de médecine vétérinaire 2004, 155(11):547-551.

16. Tassew H, Abdissa A, Beyene G, Gebre-Selassie S: Microbial flora and food borne pathogens on minced meat and their susceptibility to antimicrobial agents. Ethiopian journal of health sciences 2010, 20(3). 
17. Endale BG, Hailay G: Assessment of bacteriological quality of meat contact surfaces in selected butcher shops of Mekelle city, Ethiopia. Journal of Environmental and Occupational Science 2013, 2(2):61-66.

18. Fasanmi O, Makinde G, Popoola M, Fasina O, Matere J, Ogundare S: Potential risk factors associated with carcass contamination in slaughterhouse operations and hygiene in Oyo state, Nigeria. International Journal of Livestock Production 2018, 9(8):211-220.

19. Thrusfield M: Veterinary epidemiology. 3rd. Cambridgck e, USA: Blak Well Science Ltd 2005:225-228.

20. Beyene T, Hayishe H, Gizaw F, Beyi AF, Abunna F, Mammo B, Ayana D, Waktole H, Abdi RD: Prevalence and antimicrobial resistance profile of Staphylococcus in dairy farms, abattoir and humans in Addis Ababa, Ethiopia. BMC research notes 2017, 10(1):171.

21. Kompanikova J, Neuschlová M, Sadloňová V: Special Bacteriology - Basic Laboratory Tests. Bratislava, Slovakia; 2016.

22. Harley J, Prescott L: Bacterial morphology and staining. Laboratory Exercises in Microbiology, 5th Edition, The McGraw-Hill Companies, New York 2002:31-36.

23. Da Silva N, Taniwaki MH, Junqueira VC, Silveira N, Okazaki MM, Gomes RAR: Microbiological examination methods of food and water: a laboratory manual: CRC Press; 2018.

24. CLSI: Clinical and Laboratory Standards Institute: Performance Standards for Antimicrobial Susceptibility Testing. In: CLSI supplement M100. Wayne, PA: USA; 2018: 54-60.

25. Kebede F: Standard Veterinary Laboratory Diagnostic Manual. In: Bacteriology, Ministry of Agriculture and Rural Development Animal Health Department, Addis Ababa, Ethiopia. vol. 2; 2005: 1175.

26. Matuschek E, Brown DF, Kahlmeter G: Development of the EUCAST disk diffusion antimicrobial susceptibility testing method and its implementation in routine microbiology laboratories. Clinical microbiology and infection 2014, 20(4):0255-0266.

27. Azage $M$, Kibret M: The bacteriological quality, safety, and Antibiogram of salmonella isolates from fresh meat in retail shops of Bahir Dar City, Ethiopia. International journal of food science 2017, 2017.

28. Bersisa A, Tulu D, Negera C: Investigation of Bacteriological Quality of Meat from Abattoir and Butcher Shops in Bishoftu, Central Ethiopia. International journal of microbiology 2019, 2019.

29. Ayalew H, Berhanu A, Sibhat B, Serda B: Microbiological assessment of meat contact surfaces at abattoir and retail houses in Jigjiga town, Somali National Regional State of Ethiopia. ISABB Journal of Food and Agricultural Sciences 2015, 5(3):21-26.

30. Zerabruk K: Microbial Safety and Quality of Fresh Beef Supplied to Gullele Sub-City market, Addis Ababa. Addis Ababa University, Ethiopia; 2017.

31. Lowy FD: Antimicrobial resistance: the example of Staphylococcus aureus. The Journal of clinical investigation 2003, 111(9):1265-1273. 
32. Bitrus A, Peter O, Abbas M, Goni M: Staphylococcus aureus: A Review of Antimicrobial Resistance Mechanisms. Veterinary Sciences: Research and Reviews 2018, 4(2):43-54.

\title{
Figures
}

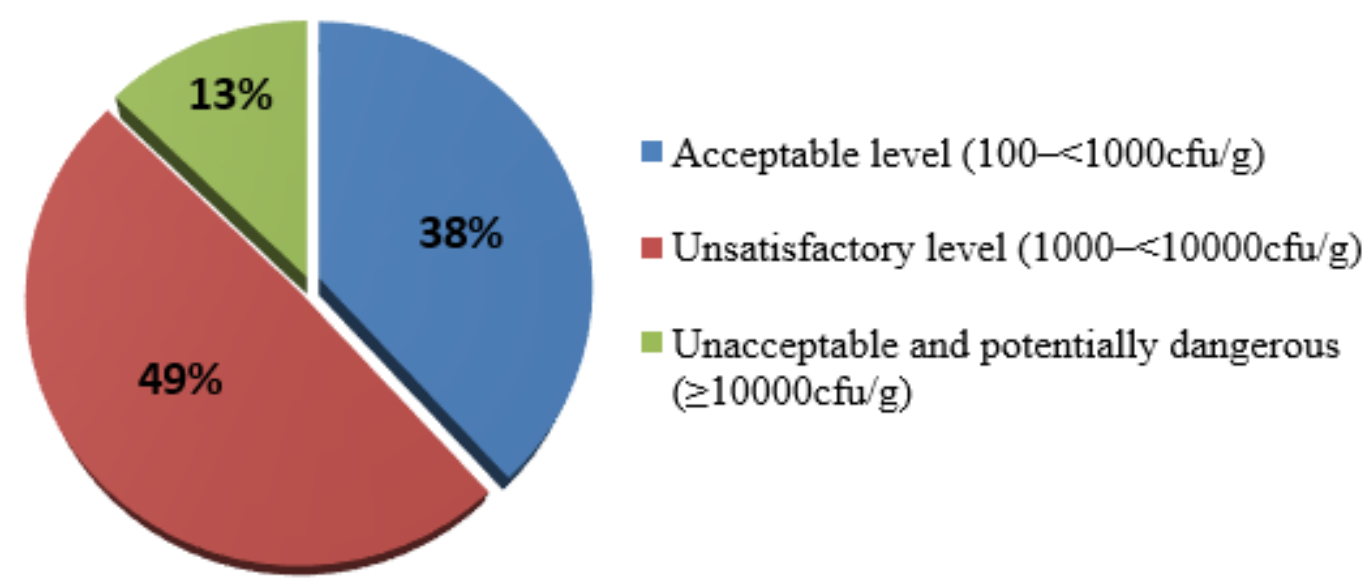

\section{Figure 1}

Microbial quality levels of butcher shops based on S. aureus count from raw beef in Bahir Dar city

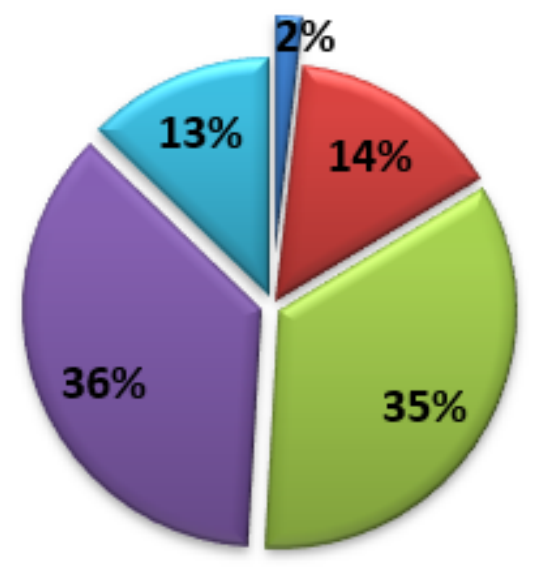

\author{
Resistant for 1 antimicrobial \\ Resistant for 2 antimicrobials \\ $\square$ Resistant for 3 antimicrobials \\ Resistant for 4 antimicrobials \\ $\square$ Resistant for 5 antimicrobials
}

\section{Figure 2}

Drug-resistance patterns of S. aureus isolates from raw beef sample in Bahir Dar city 